\title{
Neutrino oscillation results of the OPERA experiment in the CNGS beam
}

\author{
Budimir Kliček* \\ Center of Excellence for Advanced Materials and Sensing Devices, Ruđer Bošković Institute, \\ HR-10000 Zagreb, Croatia \\ E-mail: budimir.klicekeirb.hr
}

\section{Matteo Tenti}

INFN - Sezione di Bologna, viale Berti-Pichat 6/2, Bologna, Italy

E-mail: matteo.tentiabo.infn.it

\begin{abstract}
The OPERA experiment was designed to observe the appearance of tau neutrinos in the muon neutrino CNGS beam. This goal was successfully reached by observing a high purity sample of $v_{\tau}$ charged current (CC) interaction candidate events. Additionally, it was possible to isolate samples of $v_{e}$ and $v_{\mu}$ CC candidates, as well as neutral current candidate events. These four samples were used to put additional constraints on parameters of both the standard neutrino mixing model and the $3+1$ sterile model. This poster will review the methodology of measuring neutrino interactions using the OPERA apparatus and give an overview of all results on neutrino oscillations produced by the experiment. In particular, a joint analysis of $v_{\tau}$ and $v_{e}$ samples will be shown which excludes a significant fraction of the sterile neutrino phase space allowed by MiniBooNE appearance analysis.
\end{abstract}

The 21st international workshop on neutrinos from accelerators (NuFact2019)

August 26 - August 31, 2019

Daegu, Korea

\footnotetext{
* Speaker.

${ }^{\dagger}$ On behalf of the OPERA Collaboration.
} 


\section{The OPERA experiment}

The OPERA experiment [1] was designed to discover tau neutrino appearance in the predominantly muon neutrino CNGS beam [2, 3]. The OPERA detector target consisted of about 150000 stacks of lead plates and emulsion films, called Emulsion Cloud Chamber (ECC) elements (Fig. 1), embedded between scintillator planes. The detector also featured two muon spectrometers, used for momentum reconstruction of charged particles (Fig. 2).

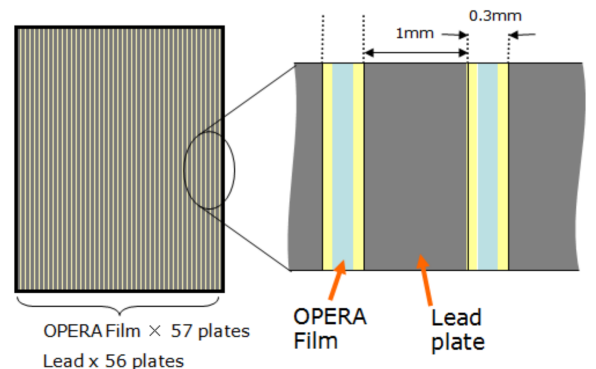

(a) Schematic view of an ECC element.

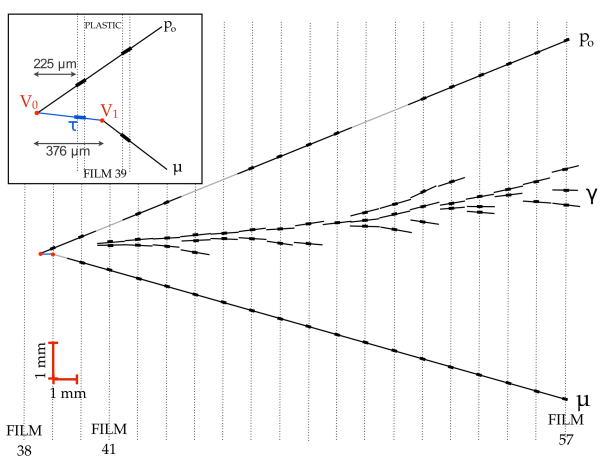

(b) Tau neutrino candidate reconstructed in the OPERA ECC. Figure taken from [4].

Figure 1: Emulsion cloud chamber.

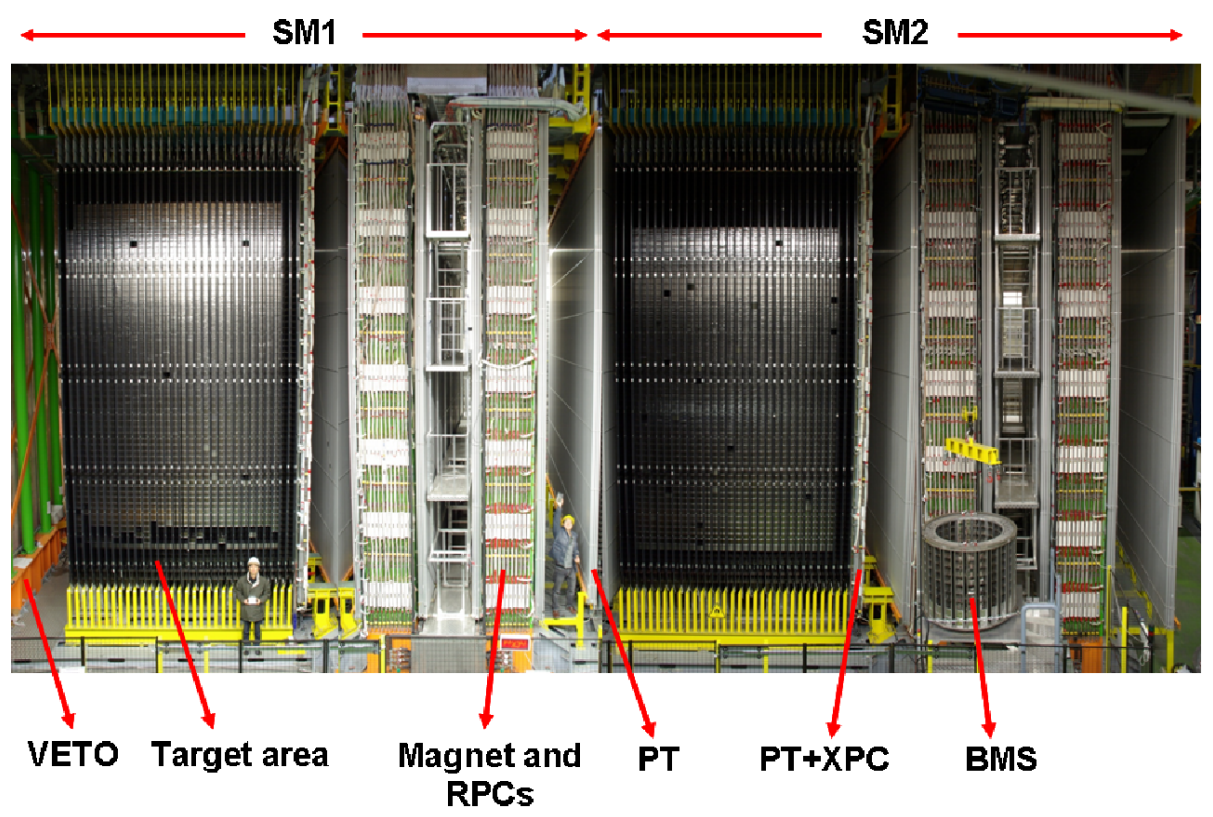

Figure 2: Side view of the OPERA detector.

Scintillator planes were used to predict the ECC element in which neutrino interaction occurred. Selected ECCs were then extracted and analysed by automatic scanning microscopes. The data taking run lasted from 2008 to 2012 with an exposure of $17.97 \times 10^{19}$ protons on target, in 
which 19505 events were recorded in the target, of which 5603 were fully reconstructed in the ECCs. The discovery of $v_{\tau}$ appearance was first published in 2015 [5], with 5 observed tau candidate events and an expected background of $0.25 \pm 0.05$ events, corresponding to a significance of $5.1 \sigma$.

\section{Neutrino oscillation results}

Final results on the $v_{\mu} \rightarrow v_{\tau}$ appearance channel were published in 2018 [6]. Using looser selection cuts, ten $v_{\tau}$ candidates were observed with an expected background of $2.0 \pm 0.4$ events (Fig. 3), corresponding to a significance of $6.1 \sigma$. Assuming maximal mixing, OPERA measured the atmospheric mass splitting in $v_{\tau}$ appearance mode to be $\left|\Delta m_{32}^{2}\right|=\left(2.7_{-0.6}^{+0.7}\right) \times 10^{-3} \mathrm{eV}^{2}$.

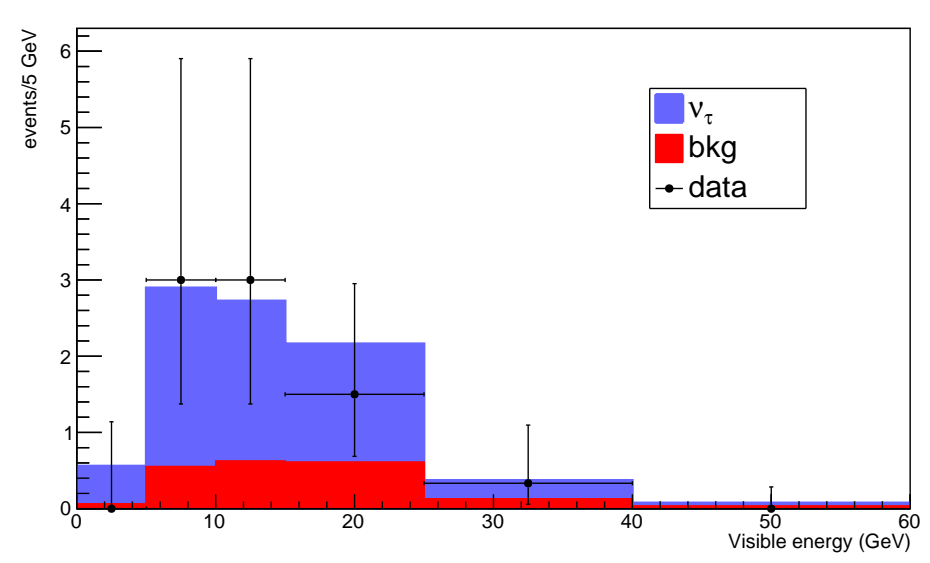

Figure 3: Energy distribution of the final sample of $10 v_{\tau}$ candidates (bullets) compared to the Monte Carlo prediction (histogram). Figure taken from [7].

The OPERA detector was also capable of measuring $v_{e}$ CC interactions, which allowed it to probe $v_{\mu} \rightarrow v_{e}$ oscillations in the CNGS beam. The expected number of $v_{e}$ candidate events is $31.9 \pm 3.2$ in case of no oscillations, and $34.3 \pm 3.4$ for standard oscillations. 35 events were observed, consistent with both cases [7].

A joint fit using $v_{\tau}$ and $v_{e}$ appearance was done assuming both the standard neutrino oscillations and the sterile $3+1$ model. In the standard model, a constraint was made on the parameters $\theta_{13}$ and $\theta_{23}$ (Fig. 4). In the sterile model, constraints on parameter pairs $\left(\sin ^{2} 2 \theta_{\mu \tau}-\Delta m_{41}^{2}\right)$ and $\left(\sin ^{2} 2 \theta_{\mu e}-\Delta m_{41}^{2}\right)$ were made (Fig. 5), which exclude recent MiniBoone result [8] with a significance of $3.3 \sigma$ [9].

Finally, a search for $v_{\mu} \rightarrow v_{\mu}$ disappearance signal has been performed. Due to the lack of a near detector, the measured $\mathrm{NC} / \mathrm{CC}$ event rate ratio was used to search for the disappearance signal. Assuming all other oscillation parameters fixed, an upper limit on atmospheric mass splitting (Fig. 6) was obtained to be $\left|\Delta m_{32}^{2}\right|<4.1 \times 10^{-3} \mathrm{eV}^{2} @ 90 \%$ C.L.

\section{Conclusions}

OPERA is the only experiment which was able to directly study all three channels of $v_{\mu}$ 
oscillations: $v_{\mu} \rightarrow v_{\tau}, v_{\mu} \rightarrow v_{e}$, and $v_{\mu} \rightarrow v_{\tau}$. Apart from the primary $v_{\tau}$ analysis, a joint fit of $v_{\tau}$ and $v_{e}$ has been performed in both standard and sterile hypothesis. Additionally, a search to $v_{\mu}$ disappearance has been performed.

\section{References}

[1] OPERA collaboration, The OPERA experiment in the CERN to Gran Sasso neutrino beam, JINST 4 (2009) P04018.

[2] G. Acquistapace et al. CERN-98-02, INFN/AE-98/05 http://proj-cngs.web.cern.ch/proj-cngs/.

[3] R. Baldy et al. CERN-SL-99-034-DI, INFN-AE-99-05 (1999). Addendum to report CERN-98-02, INFN/AE-98/05.

[4] OPERA collaboration, Evidence for $v_{\mu} \rightarrow v_{\tau}$ appearance in the CNGS neutrino beam with the OPERA experiment, Phys. Rev. D89 (2014) 051102 [1401.2079].

[5] OPERA collaboration, Discovery of $\tau$ Neutrino Appearance in the CNGS Neutrino Beam with the OPERA Experiment, Phys. Rev. Lett. 115 (2015) 121802 [1507.01417].

[6] OPERA collaboration, Final Results of the OPERA Experiment on $v_{\tau}$ Appearance in the CNGS Neutrino Beam, Phys. Rev. Lett. 120 (2018) 211801 [1804.04912].

[7] OPERA collaboration, Final results of the search for $v_{\mu} \rightarrow v_{e}$ oscillations with the OPERA detector in the CNGS beam, JHEP 06 (2018) 151 [1803.11400].

[8] MiniBooNE collaboration, Significant Excess of ElectronLike Events in the MiniBooNE Short-Baseline Neutrino Experiment, Phys. Rev. Lett. 121 (2018) 221801 [1805.12028].

[9] OPERA collaboration, Final results on neutrino oscillation parameters from the OPERA experiment in the CNGS beam, Phys. Rev. D100 (2019) 051301 [1904.05686].

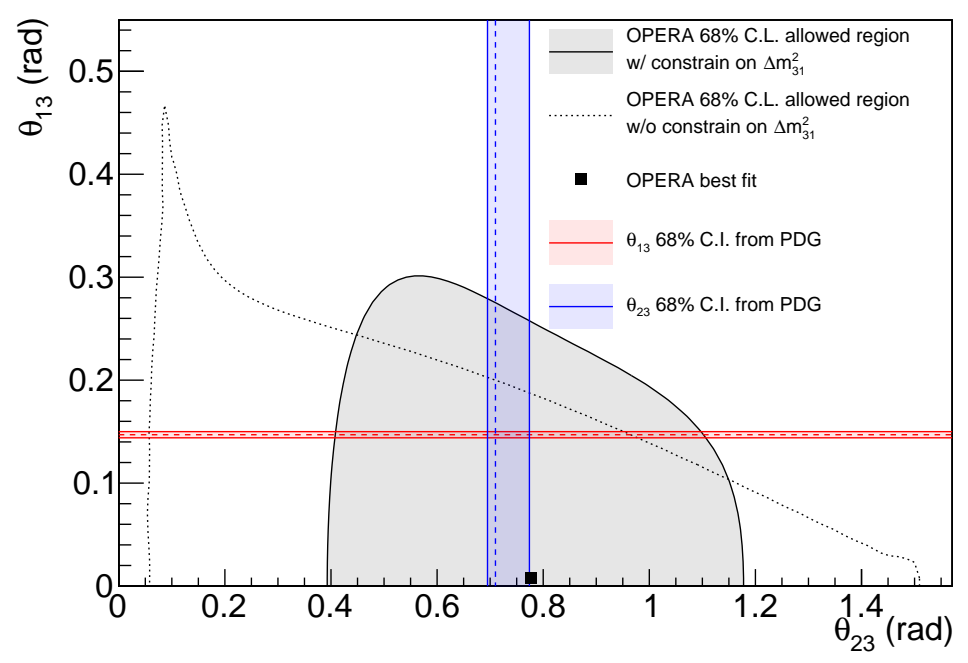

Figure 4: OPERA 68\% C.L. allowed region in the $\theta_{13}$ and $\theta_{23}$ parameter space for the normal hierarchy $(\mathrm{NH})$ of the three standard neutrino masses; assuming a prior on $\Delta m_{32}^{2}$ (filled area), and treating $\Delta m_{32}^{2}$ as a free parameter (dashed line). 

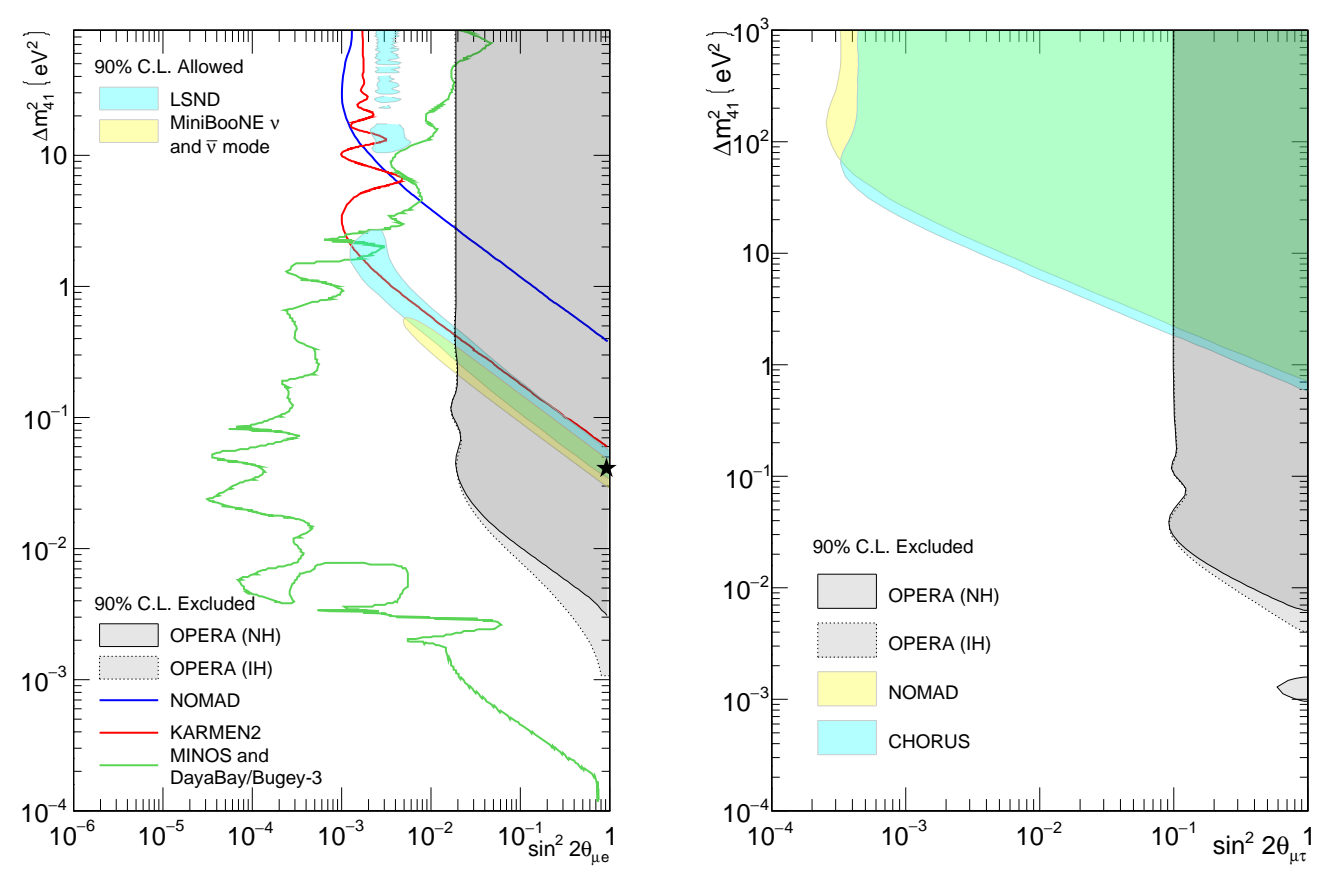

(a) The $90 \%$ C.L. exclusion region in the $\Delta m_{41}^{2}$

(b) The $90 \% \mathrm{CL}$ exclusion region in the $\Delta m_{41}^{2}$ and $\sin ^{2} 2 \theta_{\mu e}$ parameter space. The best fit of and $\sin ^{2} 2 \theta_{\mu \tau}$ parameter space.

MiniBooNE [8] is excluded by $3.3 \sigma$.

Figure 5: Sterile 3+1 model oscillation parameter constraints assuming NH and inverted neutrino mass hierarchy (IH). Figures taken from [9].

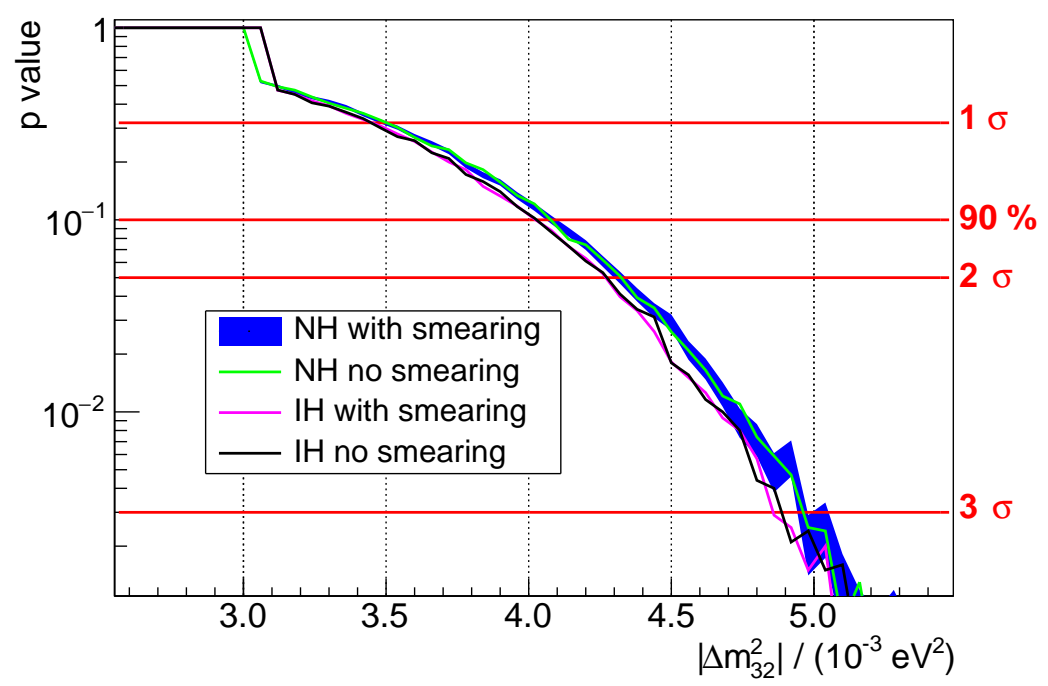

Figure 6: $p$-values as a function of $\left|\Delta m_{32}^{2}\right|$ obtained from the $v_{\mu}$ disappearance analysis, with all other oscillation parameters fixed to global fit central values. Figure taken from [9]. 